\title{
State of the art in CAR T cell therapy for CD19+ B cell malignancies
}

\author{
Matthew J. Frigault and Marcela V. Maus \\ Cellular Immunotherapy Program, Cancer Center, Massachusetts General Hospital and Harvard Medical School, Boston, Massachusetts, USA
}

\begin{abstract}
Cellular therapy for hematologic malignancies is a rapidly evolving field, with new iterations of novel constructs being developed at a rapid pace. Since the initial reports of chimeric antigen receptor T cell (CAR T cell)success in CD19+ B cell malignancies, multiple clinical trials of CAR T cell therapy directed to CD19 have led to the approval of this therapy by the FDA and the European Medicines Agency for specific indications. Despite strikingly similar efficacy, investigators at multiple centers participating in these studies have observed the nuances of each CAR T cell product, including variability in manufacturing, availability, and toxicity profiles. Here we review state-of-the-art clinical data on CD19-directed CAR T cell therapies in B cell hematologic malignancies, advances made in understanding and modeling associated toxicities, and several exciting advances and creative solutions for overcoming challenges with this therapeutic modality.
\end{abstract}

\section{Introduction}

The chimeric antigen receptor (CAR) constructs that would eventually become tisagenlecleucel (tisa-cel) and axicabtagene ciloleucel (axi-cel) were first reported in 2009. These second-generation, CD19-specific CAR T cell constructs were composed of a single-chain variable fragment $(\mathrm{scFv})$ derived from the murine anti-CD19 clone FMC63 and fused to a transmembrane domain, and the endodomains of a $\mathrm{T}$ cell costimulatory receptor $(4-1 \mathrm{BB}$ in tisa-cel and $\mathrm{CD} 28$ in axi-cel) and $\operatorname{CD} 3 \zeta(1,2)$. Both constructs were tested in vitro and in xenograft mouse models, and academic investigators soon scaled up these processes to treat patients with B cell malignancies in phase I clinical trials (reviewed in ref. 3). Although these constructs target the same epitope of CD19, seemingly minor differences in the constructs, manufacturing processes, and final cell products generated significant variability in clinical toxicity and CAR T cell kinetics in patients. Remarkably, these CD19-directed T cell products induced complete responses in patients with previously refractory or multiply relapsed B cell malignancies of different origins, including diffuse large $B$ cell lymphoma (DLBCL), chronic lymphocytic leukemia (CLL), and B cell acute lymphoblastic leukemia (B-ALL) (4-6). Since these initial reports, three pivotal studies led to FDA and European Medicines Agency approvals for the CD19-specific CAR T cell products tisa-cel and axi-cel (7-9). Class-specific toxicities include cytokine release syndrome (CRS) and immune effector cell-associated neurotoxicity syndrome (ICANS). CRS spans a spectrum of severity, from a mild flu-like syndrome with high fevers, fatigue, and

Conflict of interest: MVM is or has been a consultant for Adaptimmune, Allogene, Arcellx, Century, CRISPR Therapeutics, GSK, Incysus, Kite Pharma, MicroMedicine, Novartis, TCR2, and WindMIL; has received research funding from CRISPR Therapeutics and Kite Pharma; and holds equity in TCR2 and Century. MVM is an inventor on several patents in the field of CAR T cell therapies and ex vivo T cell culture systems. MJF is or has been a consultant for Arcellx, Kite/Gilead, Novartis, and Juno/BMS

Copyright: @ 2020, American Society for Clinical Investigation.

Reference information: / Clin Invest. 2020;130(4):1586-1594.

https://doi.org/10.1172/JCl129208. myalgias to multi-organ system failure requiring intensive supportive care such as, intubation, vasopressors, and hemodialysis (10). CAR T cell-related ICANS, previously referred to as neurotoxicity- or CAR-related encephalopathy syndrome, is a protean clinical syndrome characterized by confusion, obtundation, seizures, visual/auditory hallucinations, amnesia, expressive aphasia, and-in rare cases-potentially lethal cerebral edema $(10,11)$.

\section{Toxicities associated with CAR T cells}

The unique toxicity profile of CAR T cell therapies targeting CD19, namely CRS and ICANS, began to emerge in the first clinical trials $(4,5,12,13)$. A combination of clinical acumen and correlative immunologic studies identified the mechanisms of toxicity and led to current management strategies.

Cytokine release syndrome. CRS is a potentially life-threatening systemic inflammatory response triggered by release of proinflammatory cytokines such as IL-1, IL-2, IL-6, TNF- $\alpha$, IFN- $\gamma$, GM-CSF, MCP-1, and MIP- $1 \beta$; the frequency and severity of CRS correlate with antigen-dependent $\mathrm{T}$ cell activation and expansion (13-15). CRS has variable time to onset and can begin within the first 24 hours after CAR T cell infusion; delayed CRS has also been observed $(16,17)$. Initial symptoms of CRS include fever and tachycardia, and can progress to hypotension, hypoxia, and signs of end-organ dysfunction; CRS is managed primarily with the anti-IL-6 receptor monoclonal tocilizumab (18-21). CRS differs from a similar syndrome, cytokine storm, a steroid-responsive, antigen-independent immune activation mediated by cytokines such as TNF- $\alpha(22,23)$. CRS is a clinical syndrome mediated by antigen-specific $\mathrm{T}$ cell activation and expansion, with strong interactions with innate immune compartments mediated by the IL- 6 signaling pathway. Initial attempts to manage CRS with high-dose steroids and TNF- $\alpha$ blockade failed, leading to the first successful use of tocilizumab in refractory CRS $(13,18)$. No other agents have been approved to date for managing CAR T cell-associated CRS, but several investigators have prescribed third-line agents in the setting of 
tocilizumab- and corticosteroid-refractory CRS; these include siltuximab (anti-IL-6 antibodies), anakinra (to block IL-1), and chemotherapy ( $\mathrm{T}$ cell lytic agents, such as cyclophosphamide).

Patient- and treatment-specific factors associated with severe CRS include the use of lymphodepletion chemotherapy, higher CAR T cell dose, disease burden, and elevated baseline inflammatory markers (24). Prior CAR T cell studies that did not use lymphodepleting chemotherapy (LDC) reported lower toxicity as well as decreased efficacy compared with regimens containing it. While the effects of LDC are likely multifactorial, its use is hypothesized to increase levels of homeostatic cytokines such as IL-7 and IL-15, while also depleting inhibitory Treg populations, thereby enhancing CAR T cell expansion $(25,26)$.

ICANS and fludarabine-related neurotoxicity. ICANS has been observed with both the CD19-directed bispecific T cell engager (blinatumomab) and CD19-specific CAR T cells, and has been observed with other, non-CD19+ constructs (27). ICANS is commonly associated with, and temporally follows, CRS, and clinical laboratory findings of severe ICANS often overlap with severe CRS, including elevated C-reactive protein, ferritin, and cytopenias, suggesting a mechanistic link between CRS and ICANS (28). Initially there were concerns that CNS disease may predispose to ICANS, but recent work has suggested that the mere presence of CNS disease may not lead to increased toxicity (29). Unlike CRS, ICANS is primarily managed with high-dose steroids. Tocilizumab does not ameliorate ICANS, probably because it does not efficiently cross the blood-brain barrier (BBB); worse, tocilizumab also transiently increases circulating levels of IL-6 $(20,30)$, which may potentiate the underlying inflammatory cascade. Although MRI and electroencephalography are commonly performed during acute ICANS, little is known about the implications of various findings associated with this syndrome, such as focal areas of edema on imaging, or diffuse or frontal background slowing in $\theta$ and $\delta$ frequency ranges on electroencephalography (31). Given that severe ICANS is often associated with decreased overall survival (OS), further studies are warranted (31).

Correlative studies in samples of patients with ICANS have revealed endothelial activation through the angiopoietin (ANG)/ TIE2 axis, as well as overall disruption of the BBB, and suggest these underlying mechanisms for CAR-related ICANS (28). While ANG1 is constitutively produced by vascular (including brain) pericytes and platelets, ANG2 is stored in Weibel-Palade bodies and is released upon endothelial cell (EC) activation in the setting of inflammatory insults. Displacement of ANG1 by ANG2 causes increased EC activation and microvascular permeability (28). Patients with severe ICANS also exhibit elevated levels of inflammatory cytokines and protein and $\mathrm{T}$ cell infiltrates within the CNS, suggesting increased BBB permeability. These observations suggest that early EC activation initiates a selfperpetuating cycle of increased BBB permeability, cytokine transit, vascular pericyte stress, additional EC activation, and further increases in BBB permeability (28). More recent nonhuman primate models using a CD20-specific 4-1BB-based CAR successfully recapitulated both CRS and ICANS in rhesus macaques (32). After infusion of truncated EGFR-tagged (EGFRt-tagged) CD20-specific CARs into non-tumor-bearing primates, without LDC, robust CAR T cell expansion analogous to observations in human patients was seen. These monkeys experienced B cell aplasia as well as fever, tremor, lethargy, and ataxia, all consistent with CRS/ICANS. Supporting correlative markers included elevated serum cytokines, disproportionately high cerebrospinal fluid levels of IL-6, IL-2, GM-CSF, and VEGF, and significant accumulation of both $\mathrm{CAR}^{+}$and $\mathrm{CAR}^{-} \mathrm{T}$ cells consistent with pan-T cell encephalitis (32).

With the increasing use of fludarabine-containing regimens, clinicians should be cautious of fludarabine dosing and fluctuations in creatinine clearance, given the rising incidence of late-onset fludarabine-associated neurotoxicity (33). Initial studies of fludarabine reported neurologic symptoms 20-250 days after drug exposure that manifested as worsening visual disturbances, peripheral neuropathy, dementia, ataxia, weakness, coma, and death (34). Patients with fludarabine toxicities had findings consistent with white matter demyelination, necrosis, enlarged astrocytes and oligodendrocytes, and white matter changes evident upon MRI and autopsy (35). Elimination of fludarabine's active metabolite, F-araATP, depends on adequate renal function, rendering patients with abnormal renal function particularly susceptible to toxicities (36). Increased BBB permeability in CRS/ICANS may further predispose patients to fludarabine-related neurotoxicity (37).

MAS/HLH and the CRS spectrum. Macrophage activation syndrome/hemophagocytic lymphohistiocytosis (MAS/HLH) is a clinical syndrome associated with high fevers, hepatosplenomegaly, liver/renal dysfunction, coagulopathy, cytopenias, hyperferritinemia, and hypertriglyceridemia, with evidence of hemophagocytosis often noted upon bone marrow biopsy (38). Interestingly, these characteristics commonly occur in patients experiencing severe CRS, with considerable overlap of cytokine profiles including elevated levels of IFN- $\gamma$, IL-6, and IL2RA $(39,40)$. Although rare, fatal cases of MAS/HLH have been observed in CAR T cellrelated CRS/ICANS. Unfortunately, standard NOD-SCID $-\gamma$ chain receptor-knockout (NSG) mouse models failed to predict or recapitulate CRS and ICANS, partly because of the NOD genetic background, which reduces function of the innate immune system (41). Work by Giavridis et al. (42) and Norelli et al. (43) used SCID-beige and humanized-SGM3 mouse models, respectively, which partly recapitulated much of the clinical syndrome of CRS and ICANS (Figure 1). These data demonstrated that although $\mathrm{T}$ cells are the primary source of both IFN- $\gamma$ and GM-CSF, myeloid cells are the primary producers of inflammatory factors such as IL-6, IL-1, and inducible NOS, which play key roles in the pathogenesis of CRS $(42,43)$. As excessive macrophage activation appears to be a shared characteristic in both severe CRS and ICANS, targeting the MAS pathway may ultimately yield therapeutic benefits for both clinical syndromes. Additional studies have demonstrated GM-CSF's potential role in CRS (44), and clinical trials targeting multiple aspects of the MAS pathway are likely to open in the coming year.

Grading and difficulty of cross-trial comparisons. Early clinical trials using CAR T cell therapy lacked a unified grading system for CRS and ICANS. Although early use of a modified Common Terminology for Criteria for Adverse Events (CTCAE v4.03) was attempted, it became clear that standard CTCAE grading was insufficient to truly capture CAR T cell-related toxicities. Thus, a published consensus among early investigators incorporated clin- 


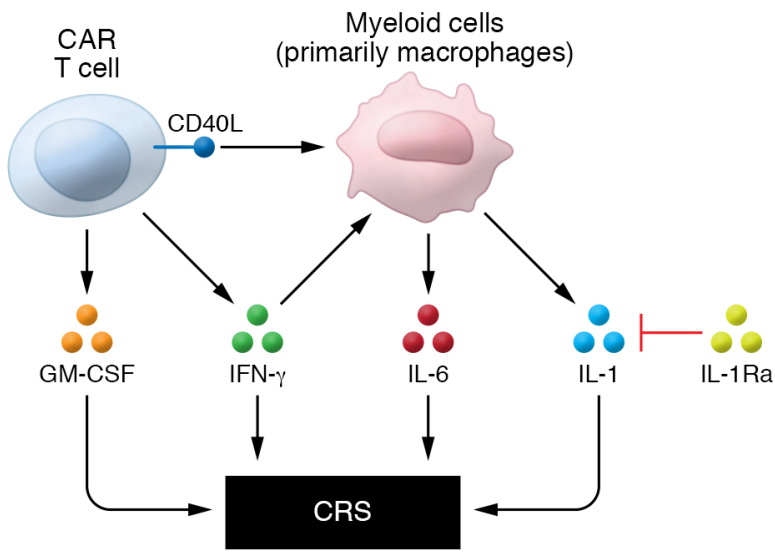

Figure 1. Summary of mechanisms discovered in two animal models designed to recapitulate cytokine release syndrome.

ical factors such as hypoxia and hypotension in combination with organ-specific CTCAE grading (16). In parallel, investigators at the University of Pennsylvania developed a CRS grading scale for their CD19-directed CAR T cell trials focusing on clinically relevant factors and interventions, including need for hospitalization and varying degrees of organ-specific toxicities (45). Unlike Lee et al. (16), the Penn criteria defined grade 3 CRS as any organ dysfunction requiring hospitalization, including grade 4 liver function abnormalities, grade 3 creatinine, coagulopathy requiring blood products, hypotension treated with multiple fluid boluses or lowdose pressors, and hypoxia requiring supplemental oxygen, leading to more severe grading $(10,17)$. Separately, investigators at Memorial Sloan Kettering Cancer Center developed criteria using duration of intervention for hypoxia and/or hypotension, while investigators at MD Anderson Cancer Center developed the CARTOX criteria, modifying Lee et al. (16) slightly by including organ toxicities in the definition of grade 1 CRS $(46,47)$.

In an attempt to harmonize these various approaches, an expert group comprising academic centers and industry partners convened at a 2018 meeting supported by the American Society for Transplantation and Cellular Therapy. These new criteria defined CRS as the presence of fever with/without hypotension and/or hypoxia. Grading depends on the degree of vasopressor and respiratory support, with clear delineations based on the type of intervention. End-organ toxicities were removed from the process of grading severity, as such toxicities were felt to be managed symptomatically in accordance with standard medical practices. ICANS was defined as "a disorder characterized by a pathologic process involving the central nervous system following any immune therapy that results in the activation or engagement of endogenous or infused $\mathrm{T}$ cells and/or other immune effector cells," with the consideration that this system could be applied to adverse effects of immune effector cell-engaging therapies beyond CAR T cells. Additionally, a scoring algorithm was devised to include an element for assessing receptive aphasia, which is now termed the Immune Effector Cell-Associated Encephalopathy (ICE) score (10). Adopting these consensus criteria in future studies and in clinical practice will enable cross-study comparisons of new and existing immune effector cell therapies.

\section{Summary of current FDA-approved indications for CD19-directed CAR T cell therapy}

There are two CD19-directed CAR T cell products approved and one nearing approval for various indications that originate from $\mathrm{B}$ cell malignancies: tisagenlecleucel (tisa-cel) for pediatric B-ALL and large cell lymphoma, axicabtagene ciloleucel (axi-cel) for large cell lymphoma, and, nearing approval, lisocabtagene maraleucel (liso-cel) for large cell lymphoma. The results of each registration trial are summarized in Table 1 and described in more detail below.

Tisa-cel for B-ALL. The FDA approved the first CAR T cell therapy in August 2017 for relapsed/refractory pediatric and young adult B-ALL up to 25 years of age based on the phase II, global ELIANA study (9). In this trial, 75 patients were infused following LDC (fludarabine $30 \mathrm{mg} / \mathrm{m}^{2}$ i.v. for 4 days, cyclophosphamide $500 \mathrm{mg} / \mathrm{m}^{2}$ i.v. for 2 days). The overall complete remission rate within 3 months was $81 \%$; all patients in remission were negative for minimal residual disease (MRD) as scored by flow cytometry. Impressively, the event-free and OS rates of these patients were $50 \%$ and $76 \%$, respectively, at 12 months, with a median follow-up of 13.1 months. Forty-six percent of patients experienced grade $3+$ CRS, based on the grading criteria developed at the University of Pennsylvania (45), while only $13 \%$ experienced grade $3+$ neurotoxicity. In this cohort, the median number of prior therapies was 3 (range $1-8$ ), with $61 \%$ of patients having relapsed after a prior allogeneic stem cell transplant (SCT). Responses were equivalent regardless of prior SCT, presence of high-risk mutations such BCR-ABL1, MLL, hypoploidy, or BCR-ABL1-like gene signatures. Because there was no correlation between cell dose and CAR T cell expansion, as measured by mean area under the curve from day 0 to day $28\left(\mathrm{AUC}_{0-28 \mathrm{~d}}\right)$ and maximum transgene level $\left(\mathrm{C}_{\max }\right)$, a wide target cell dose of $0.2 \times 10^{6}$ to $5.0 \times 10^{6} \mathrm{CAR}^{+}$viable $\mathrm{T}$ cells was allowed for commercial tisa-cel in B-ALL. The median persistence of CAR T cells was 168 days in responding patients. Most importantly, there was no difference in OS with or without censoring at the time of allogeneic SCT, with only 8 of 75 patients undergoing later allogeneic SCT after CAR T cell therapy (9).

In subsequent analyses of this trial, patients with B cell recovery within 6 months of infusion experienced earlier loss of CAR $\mathrm{T}$ cell persistence, as determined via transgene copy number (48). Furthermore, no relationships were detected among clinical response, safety, expansion, and CD4/CD8 ratio of infused product (48). Longer-term follow-up demonstrated similarly impressive response rates, with relapse-free and OS rates at 18 months of $66 \%$ (95\% CI, 52\%-77\%) and $70 \%$ (95\% CI, 58\%-79\%), respectively (49). Of the responders, 19 patients relapsed; 14 of 19 patients exhibited loss of CD19 in their tumor despite ongoing B cell aplasia, a phenomenon previously attributed to hemizygous deletions spanning the CD19 locus and de novo frameshift and missense mutations in extracellular portions of CD19 as well as CAR transduction of leukemic blasts leading to epitope masking $(50,51)$. As cellular immune responses against the murine portions of the anti-CD19 scFv (FMC63) have been demonstrated (52), subsequent generations of $\mathrm{CD} 19^{+} \mathrm{CAR} \mathrm{T}$ cells may utilize humanized versions of the anti-CD19 scFv. Preliminary data for such humanized anti-CD19 CAR T cell therapy (CTL119) have demonstrated efficacy in relapsed/refractory disease after failure of murine-derived (FMC63) anti-CD19 CAR T cells $(53,54)$. 


\section{Table 1. Summary of efficacy and toxicity from advanced CD19-directed CAR T cell therapies}

\section{Tisagenlecleucel}

(95)

Anti-CD19-4-1BB-CD3ל

Construct

Follow-up, months

Median prior therapies

Overall response

Median OS, months

PFS at 2 years, \%

All patients

In CR

Grade 3-4 AEs

CRS grading scale used

Treatment locale

Approval status

24

3

$54 \%$ ORR, $40 \%$ CR in $C R$

Not

$23 \%$ CRS,

Inpatient or outpatient

Axicabtagene ciloleucel

(56)

Anti-CD19-CD28-CD3C

10.3 , not reached for patients

27.1

3

$83 \%$ ORR, $58 \%$ CR

Not reached

Lisocabtagene
(69)

Anti-CD19-4-1BB-CD3ל

12

73\% ORR, 53\% CR

Not reached

FDA-approved for pediatric AL

and adult R/R DLBCL

AEs, adverse events; CR, complete response; NT, neurotoxicity; ORR, overall response rate; OS, overall survival; PFS, progression-free survival; PMBL, primary mediastinal B cell lymphoma; R/R, relapsed/ refractory.

toxicity, $11 \%$ of patients experienced grade 3+ CRS (per Lee et al., ref. 16), while $32 \%$ experienced grade $3+$ neurotoxicity. Two treatment-related deaths from HLH and cardiac arrest were reported (56).

Given these impressive data, two groups independently sought to describe the off-protocol, commercial "real-world" experience of treatment with axi-cel in patients who were eligible based on the prescribing label, but who may or may not have met the stricter eligibility requirements of the registration trial. At the 2018 American Society of Hematology (ASH) Annual Meeting, Nastoupil et al. (59) and Jacobson et al. (60) reported on a collective 269 patients who received axi-cel in October 2017. These groups reported objective response rates of $79 \%$ and $71 \%$, respectively, and complete response rates of $50 \%$ and $44 \%$ respectively, similar to rates reported

Axi-cel for DLBCL. In October 2017, axi-cel was the second CAR T cell product to be approved in the United States. The approval was based on a single-arm, phase II, multicenter registration trial (ZUMA-1) for relapsed or refractory large B cell lymphoma after two or more lines of systemic therapy, including DLBCL not otherwise specified, primary mediastinal large B cell lymphoma, high-grade B cell lymphoma, and DLBCL arising from follicular lymphoma (55). Of 101 assessable patients who received axi-cel after LDC (fludarabine $30 \mathrm{mg} / \mathrm{m}^{2}$ i.v. and cyclophosphamide $500 \mathrm{mg} / \mathrm{m}^{2}$ i.v. for 3 days), $83 \%$ had an objective response, including a complete response rate of $58 \%$. At the time of data cutoff, $39 \%$ of patients had ongoing responses with a median follow-up of 27.1 months. Interestingly, $39 \%$ of patients who had either a partial response or stable disease at 1 month eventually converted to a complete response by 6 months (56). Of the study population, $39 \%$ had complete responses, and importantly, in this group, the median duration of response had not been reached after 2 years. Notably, in this disease, some consider 2 years of complete remission from chemotherapy as representing cure (57) and no longer requiring routine follow-up imaging. CD19 positivity was not required for eligibility, as the frequency of preexisting CD19- disease was considered negligible. Retrospective analysis of CD19 expression by immunohistochemistry did not predict response, likely owing to decreased sensitivity of immunohistochemistry compared with CAR scFv recognition; however, a subset of patients who relapsed did exhibit $\mathrm{CD}^{-} 9^{-}$disease only at relapse (58). Responders demonstrated higher CAR T cell expansion $\left(\mathrm{AUC}_{0-28 \mathrm{~d}}\right.$ and $\left.\mathrm{C}_{\max }\right)$ than nonresponders, and although very low levels of axi-cel could be detected in responders at day +180 , only three patients with ongoing complete remissions had detectable CAR T cells at 24 months (55). The role of expansion and persistence thus seems to differ from that seen with tisa-cel; this may be related to their different costimulation domains. Regarding by the pivotal ZUMA-1 study (61). Interestingly, when Nastoupil et al. and Jacobson et al. examined patient populations based on ZUMA-1 eligibility criteria, only $51 \%$ and $40 \%$ met ZUMA- 1 eligibility, respectively, with most ineligibility linked to performance status, renal dysfunction, and cardiac dysfunction $(59,60)$. Unlike tisa-cel, axi-cel is prescribed using fixed weight-based dosing of $2 \times 10^{6} \mathrm{CAR}^{+} \mathrm{T}$ cells $/ \mathrm{kg}$, with a maximum dose of $2 \times 10^{8} \mathrm{CAR}^{+}$ $\mathrm{T}$ cells. Correlative studies from the ZUMA-1 study indicated that the $\mathrm{CD} 4 / \mathrm{CD} 8$ ratio of infused product was not predictive of response; however, more recent work (62) demonstrated that preinfusion polyfunctionality of the $\mathrm{T}$ cell compartment was associated with improved clinical outcomes, albeit at the expense of increased toxicity. CD19 loss and downregulation of CD19 and other B cell antigens, as well as PD-L1 upregulation, may underlie possible tumor resistance and/or relapse $(60,63,64)$.

Tisa-cel for DLBCL. The international, phase II, JULIET study (8) led to the May 2018 approval of tisa-cel for adult patients with relapsed or refractory large B cell lymphoma after two or more lines of systemic therapy, including DLBCL not otherwise specified, high-grade B cell lymphoma, and DLBCL arising from follicular lymphoma. Ninety-three patients infused with tisa-cel after LDC (fludarabine $25 \mathrm{mg} / \mathrm{m}^{2}$ i.v. and cyclophosphamide $250 \mathrm{mg}$ / $\mathrm{m}^{2}$ i.v. for 3 days) were evaluable for efficacy, with a median time from infusion to data cutoff of 14 months (8). The best objective response rate was $52 \%$ (95\% CI, $41 \%-62 \%)$, with $40 \%$ of all patients achieving a complete response. As in the ZUMA-1 study (61), responses occurred across all subgroups regardless of molecular subtype, prior transplant, and double/triple-hit disease (8). The median duration of response for the $35 \%$ of patients who had ongoing responses at the data cutoff, including $34 \%$ with ongoing complete responses, had not been reached at 19.3 months of follow-up (65), again indicating the potential curative nature of CAR $\mathrm{T}$ cell therapy. Although CD19 positivity was required per proto- 
col, retrospective analysis of CD19 expression levels did not indicate that expression level correlated with response. Unlike with axi-cel, similar in vivo expansion $\left(\mathrm{C}_{\max }\right.$ and $\left.\mathrm{AUC}_{0-28 \mathrm{~d}}\right)$ was observed in responders and nonresponders and persistent CAR transgene levels were observed for up to 2 years in most patients with durable responses. Like in ELIANA, activity was seen across a wide dose range, leading to the FDA-approved dosage of $0.6 \times 10^{8}$ to $6.0 \times 10^{8}$ $\mathrm{CAR}^{+} \mathrm{T}$ cells. Twenty-two percent of patients experienced grade $3+$ CRS (according to the Penn Criteria [ref. 45]), while 12\% experienced grade $3+$ neurotoxicity. No deaths were attributed to CAR T cell treatment, CRS, or neurotoxicity. Unlike the ZUMA-1 study (61), JULIET allowed for bridging therapy between leukapheresis and CAR T cell infusion (8). Thus, a subset of 7 patients achieved a complete response to salvage chemotherapy; this response aligned with response rates predicted by an international, multicohort, retrospective, non-Hodgkin lymphoma (NHL) research study (SCHOLAR-1) (66), and proceeded with tisa-cel infusion. Despite absence of radiographic disease, tisa-cel expanded in all 7 patients, and transgene levels were detectable for more than 2 years, with 5 of 7 patients remaining disease-free at $>12$ months ( 8 , 67); these data indicate that a large burden of antigen is not necessary for the CAR T cells to expand and persist in patients. Additional data supported tisa-cel's use in isolated, secondary CNS disease, again suggesting that active systemic disease may not be required for tisa-cel expansion and activity (29), demonstrating that CAR T cell therapy may penetrate disease compartments that are considered sanctuaries from standard chemotherapy and antibody therapy.

Liso-cel for DLBCL. Another promising CD19-specific construct in development is lisocabtagene maraleucel (liso-cel), previously known as JCAR017. Like axi-cel and tisa-cel, liso-cel is a second-generation CD19-specific CAR T cell construct using the FMC63-derived scFv. Although it shares a 4-1BB costimulatory domain with tisa-cel, liso-cel's structure uses a modified IgG4 hinge and transmembrane domain rather than CD8 hinge and transmembrane domains. Its predefined manufacturing process of $1: 1 \mathrm{CD}^{+} / \mathrm{CD}^{+} \mathrm{CAR} \mathrm{T}$ cells is based on preclinical work suggesting synergistic enhancement of antitumor activity by administration of a defined ratio of $\mathrm{CD}^{+}$to $\mathrm{CD}^{+}$cells in a xenograft model of Burkitt lymphoma (68). Data presented at ASH's 2019 annual meeting indicate that when liso-cel was administered across three dose levels in 268 patients, the objective response rate was $73 \%$, with a best overall complete response rate of $53 \%$. The median progression-free survival of all patients was 6.8 months, with a median OS of 19.9 months. CRS (graded according to Lee et al., ref. 16) occurred in $42 \%$ of patients, and $2 \%$ exhibited grade $3+$ severe CRS. Neurotoxicity was also detected in about $30 \%$ of patients, with $10 \%$ having grade $3+$ or higher (69).

\section{Moving beyond currently approved indications}

Earlier lines of therapy or combination with SCT. There is substantial motivation to move CAR T cell therapy earlier in the patient treatment course, rather than focusing on end-stage disease. Relapsed/ refractory lymphoma is currently defined as chemotherapyrefractory disease following two lines of therapy or relapsed following autologous SCT. Data from the phase III ORCHARRD trial (70), the largest second-line trial using rituximab- and anthracy- cline-based first-line regimens, indicated 2-year progression-free survival and os of $24 \%-26 \%$ and $38 \%-41 \%$, respectively; only $33 \%-37 \%$ of patients completed autologous SCT, highlighting a substantial need in this patient subset. Studies are under way to investigate axi-cel, tisa-cel, and liso-cel in earlier lines of therapy for aggressive lymphomas, including for first-line DLBCL (ZUMA-12/axi-cel, NCT03761056, ClinicalTrials.gov) and second-line DLBCL versus standard-of-care autologous SCT (ZUMA7/axi-cel, NCT03761056; Belinda/tisa-cel, NCT03570892; and TRANSFORM/liso-cel, NCT03575351). Additional studies are evaluating the use of CAR T cells in earlier-line therapy after first relapse or $\mathrm{MRD}^{+}$pediatric and adult leukemia (NCT03628053).

Various groups have attempted to incorporate CAR T cell therapy after autologous SCT; in all instances, CAR T cell infusion followed preconditioning chemotherapy and stem cell infusion. The initial studies used first-generation (CD3ל only) and secondgeneration (CD28ל) constructs introduced into T cells with differing viral and transposon-based transduction methods (71). These studies included patients with various B cell NHL (B-NHL) subtypes with either active disease or undetectable disease by imaging, and reported 1-year progression-free survival of $75 \%(71,72)$. Sauter et al. (73) recently reported a larger series of poor-risk, chemosensitive relapsed and refractory B-NHL patients who underwent CAR T cell therapy after SCT. Of the 15 patients, $67 \%$ experienced severe neurotoxicity, with a 2-year progression-free survival of $30 \%$. Interestingly, the proportion of naive-like $\left(\mathrm{CD} 45 \mathrm{RA}^{+} \mathrm{C}-\right.$ $\mathrm{CR}^{+}$) CAR T cell populations in the infused product was inversely correlated with progression-free survival (73).

CD19-directed CAR T cell therapy beyond adult high-grade lymphomas. Although current approvals of CAR T cell therapies only encompass pediatric/young adult B-ALL and adult DLB$\mathrm{CL}$, success was originally achieved in indolent lymphomas and adult B-ALL, but these indications have yet to obtain FDA approval (4-6). Axi-cel is currently being studied in multiple disease settings, including follicular and marginal-zone lymphomas (ZUMA-5/NCT03105336), mantle cell lymphoma (MCL) (ZUMA2/NCT02601313) (74), chronic lymphocytic leukemia (CLL) (ZUMA-8/NCT03624036), and adult (ZUMA-3/NCT02614066) and pediatric/young adult ALL (ZUMA-4/NCT02625480). Recently presented data from ZUMA-2 $(n=28), \mathrm{KTE}-\mathrm{X} 19$ in MCL, appeared promising with an overall response rate of $86 \%$, complete response rate of $57 \%$, and ongoing responses in $83 \%$ of patients at 12 months. Tisa-cel is also under investigation for pediatric NHL in a multicenter phase II protocol (BIANCA, NCT03610724), for follicular lymphoma in a multicenter phase II study (ELARA, NCT03568461), for relapsed/refractory adult B-ALL in a randomized, phase III study comparing CAR T cell therapy with blinatumomab or inotuzumab (Oberon/NCT03628053), and for primary CNS lymphoma in a pilot single-center study (NCT04134117). Although liso-cel has yet to obtain FDA approval, additional studies involving CLL/small lymphocytic leukemia as well as MCL are ongoing (NCT03331198, NCT02631044). Other studies are examining the role of combinatorial therapies to improve on already impressive CAR T response rates. Axi-cel is currently being studied in combination with PD-L1 blockade (ZUMA-6, atezolizumab, NCT02926833), 4-1BB agonistic antibodies (ZUMA-11, utomilumab, NCT03704298), and rituximab and/or lenalido- 
A

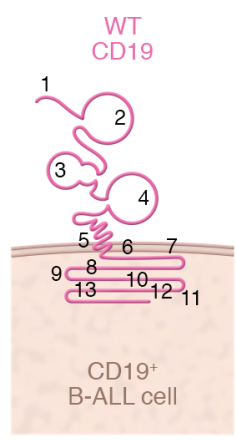

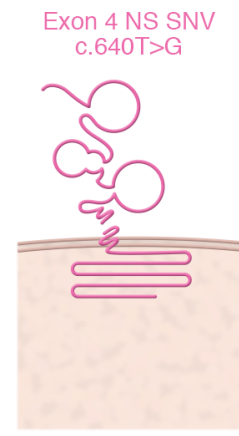

B

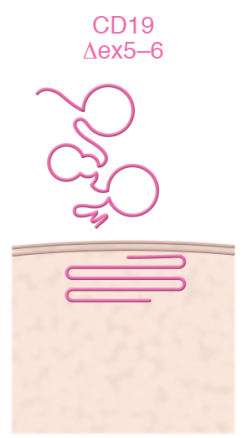

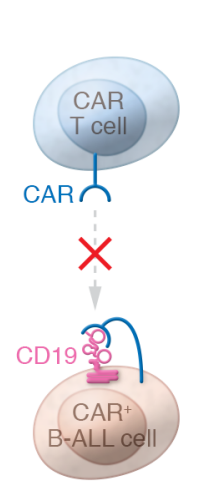

C

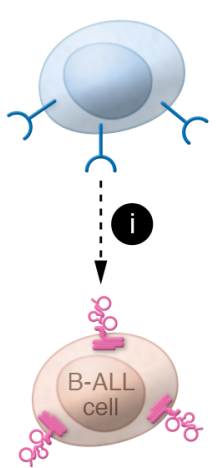

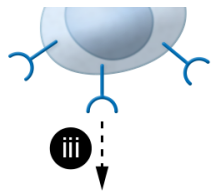

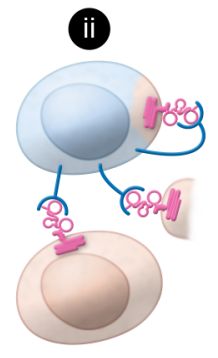

Figure 2. Examples of mechanisms underlying CD19- loss in B cell malignancies. (A) Wild-type CD19 and observed frameshift mutations that result in lack of surface expression of the CD19 molecule in B cell leukemia. (B) CAR-transduced B cells with self-binding ("masking") of CD19 by the CAR expressed on the cell surface of leukemic B cells. (C) Trogocytosis, in which CAR T cells "rip off" the CD19 molecules from the leukemic blasts by membrane transfer, resulting in lowered expression of CD19 by the leukemic blasts. In (i), CAR T encounters B-ALL cell; in (ii), CAR T has trogocytosed bits of membrane-bound CD19 from the B-ALL cell onto the CAR T cell, and some of the CD19 on the CAR T cell is masked; in (iii), the CAR T cell with membrane-bound CD19 may become susceptible to lysis by another CAR T cell.

mide (ZUMA-14, NCT04002401). Early results demonstrated that combining atezolizumab with axi-cel resulted in a more than 20 -fold higher CAR T cell expansion without significant increases in CAR T cell-related toxicities (75). Tisa-cel is being evaluated in combination with ibrutinib (NCT03876028) and with the PD-1 inhibitor pembrolizumab (Portia, NCT03630159). Finally, liso-cel is being studied in combination with the PD-L1 inhibitor durvalumab and the novel cereblon-modulating agent CC-122 (PLATFORM, NCT03310619).

\section{Mechanisms of tumor resistance}

Despite dramatic treatment responses in a large subset of patients with otherwise refractory disease, upwards of $50 \%$ of patients eventually relapse after CD19-directed CAR T cell therapy. Further research is therefore warranted to determine mechanisms of disease resistance and to evaluate potential approaches to improve overall response rates and response durability. The factors underlying disease resistance likely fall into three categories: poor $\mathrm{T}$ cell fitness/expansion, loss of the target antigen, and, most recently, tumor mutations that lead to resistance to CAR T cell-mediated killing (76).
Poor $T$ cell fitness or expansion. Porter et al. (45) first described the role of CAR T cell expansion and persistence as correlating with treatment responses. Among patients who achieved complete response, median transgene expansion was more than 73,000 copies/ $\mu$ g (assayed via PCR), while nonresponders displayed minimal expansion, with a median of 420 copies/ $\mu$ g; peak transgene copy number was correlated with response. In the same patient series, CAR T cells persisted longterm, as assessed via flow cytometry and quantitative PCR, and all responding patients had ongoing $B$ cell aplasia, suggesting functional persistence of their CD19-directed CAR (45). Fraietta et al. (77) later established that CAR T cells from patients who achieved complete response were enriched for the expression of memory-related genes, including IL-6/STAT3 signatures, whereas nonresponders upregulated expression programs involved in effector differentiation and exhaustion. These findings were further validated by the report that remission was associated with an elevated frequency of $\mathrm{CD} 8{ }^{+} \mathrm{CD} 27^{+} \mathrm{CD} 45 \mathrm{RO}^{-}$ memory-like $\mathrm{T}$ cells before CAR T cell manufacturing (77). Prior work had demonstrated that pretreatment with ibrutinib, a BTK/ITK inhibitor, improved expansion of CD19-directed
A

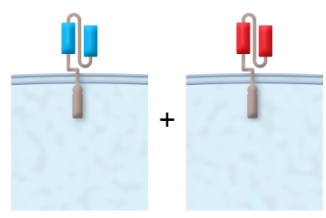

Pooled CAR populations
B

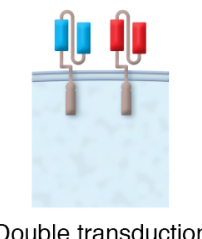

C

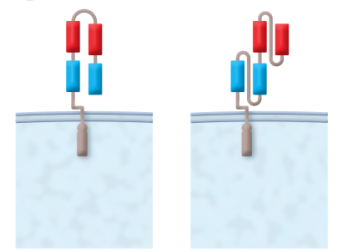

Tandem CARs
D

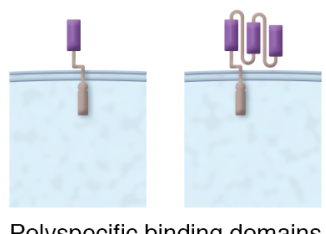

E

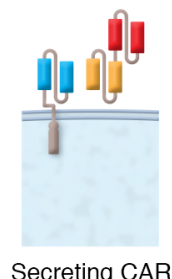

Figure 3. Summary of strategies for targeting multiple antigens with CAR T cells. (A) Pooled CAR populations: Two cell populations are transduced with two different CARs, each with a single specificity (red and blue); these two cell products are then pooled or coinfused into the patient. (B) Double transduction: One cell population is cotransduced with two CARs, each with a single specificity (red and blue). (C) Tandem CARs: Two different binding specificities (red and blue) are molecularly linked in tandem and fused to a single transmembrane/signaling domain. The specificities can be combined in different formats to achieve productive binding of each. (D) Polyspecific binders: A cross-reactive CAR (purple) binds with two different specificities. These can be linked together in tandem to form multimeric binding domains. (E) Secreting CARs: The CAR has one specificity (blue) and a second binder is secreted from the CAR (red). This can also take the form of a T cell engager (yellow) if the secreted form has a bispecific format. 
CAR $\mathrm{T}$ cells in association with deceased expression of the immune checkpoint PD-1 (78). Additional work is under way using coculture with PI3K inhibitors during CAR T cell manufacturing to enrich for a memory-like phenotype and improved functional persistence of CAR T cells (79). Taken together, these data suggest that $\mathrm{T}$ cell fitness drives CAR $\mathrm{T}$ cell function, and that $\mathrm{T}$ cell fitness is amenable to improvement with small-molecule drugs.

Given that patients with hematologic malignancy may not recover $\mathrm{T}$ cell fitness, because of either disease or prior therapies, CAR T cell approaches using allogeneic CAR T cells are under development. With allogeneic $\mathrm{T}$ cell products, the endogenous $\mathrm{T}$ cell receptor needs to be removed to prevent graft-versus-host disease in the recipient. To this end, gene-editing approaches using TALENs or CRISPR/Cas must be used to knock out the $\mathrm{T}$ cell receptor, which can be done in one or two steps with gene transfer of the CAR transgene. Early clinical investigations of the use of allogeneic CAR $\mathrm{T}$ cell therapy for relapsed/refractory B-ALL (UCART19) have reported overall complete response rates of $88 \%$, with $86 \%$ of responses having no MRD detectable by flow cytometry or quantitative PCR. However, although initial responses are promising, subsequent host-mediated rejection of these allogeneic products may limit efficacy or have other unforeseen consequences (80).

Loss of the target antigen. To date, multiple mechanisms of antigen loss have been reported in patients who relapse after CAR $\mathrm{T}$ cell therapy, underscoring the strong selection pressure exerted by this therapy. Frameshift mutations leading to CD19 transmembrane domain loss were detected in a subset of previously $\mathrm{CD} 19^{+}$patients with $\mathrm{CD} 19^{-}$disease relapse after CAR T cell therapy (Figure 2 and ref. 81). In addition to harboring genomic alterations, a series of $\mathrm{CD}^{-}{ }^{-} \mathrm{B}-\mathrm{ALL}$ relapses resulted from splice variants, with loss of the exon encoding the epitope targeted by FMC63 (50) or loss of the anchoring transmembrane domain (Figure 2A). In immature B cell malignancies, Gardner et al. reported a conversion of acute lymphoblastic to acute myeloid leukemia in a patient with mixed-lineage leukemia; this genetically identical - but phenotypically myeloid - relapse was impervious to CD19 targeting (82). "Loss" of CD19 antigen expression has also been observed in the context of antigen masking due to CAR transduction into leukemic blasts (51) (Figure 2B). CAR-transduced blasts effectively mask the target epitope from external CAR T cell killing through self-binding of CD19 on the cell surface (51). Although retrospective analysis identified several patients with CAR-transduced B cells in their preinfusion products, only one had CD19relapse of disease, indicating that this event is quite rare. Finally, in in vitro and animal models, CARs have been shown to induce reversible antigen loss through trogocytosis, in which the target antigen is transferred to CAR T cells during establishment of an immune synapse (Figure 2C). This transfer of target antigen led to a decrease in target density on tumor cells as well as an increase in fratricide and subsequent $\mathrm{T}$ cell exhaustion (83); this mechanism of relapse has not been definitively observed in patients.

Analogous to historical data with antimicrobial therapy, where serial targeting of a single pathway can lead to resistance, approaches to target multiple surface antigens simultaneously are under way with the hypothesis that these are more likely to avoid antigen relapse (Figure 3). Although dual targeting with two separately transduced CAR T cell populations is possible (Figure 3A), transduction of a single cell population with pooled vectors (Figure $3 \mathrm{~B}$ ) or a bicistronic vector expressing dual-antigen specificity (Figure 3C) had higher efficacy in animal models of antigen ${ }^{-}$relapse (84, 85). Based on early data from a phase I study of a bispecific CD19/ CD20 second-generation CAR construct, the objective response rate was $91 \%$, with an $82 \%$ complete response rate at the recommended phase II dose and no relapses to date, although follow-up is limited (86). Other approaches using multiple scFvs attached to a single intracellular signaling domain allow for a reduced transgene size while providing multi-antigen specificity; for example, such tandem CARs targeting CD19/CD22, CD19/CD37, and CD19/CD79b are currently under investigation (87-89). Although $\mathrm{scFv}$ has been the primary binding moiety employed in CAR T cell constructs to date, alternative binders such as camelids, adaptor proteins, and modified ligands/receptors are also under clinical development. Naturally occurring binders may achieve the same dual-antigen targeting without extensive modification to $\mathrm{scFv}$ orientations and with reduced immunogenicity (Figure 3D). This approach was validated in a recent study of IL13Ra2 for glioblastoma, and similar approaches include using a proliferation-inducing ligand (APRIL) as well as a trimeric APRIL (TriPRIL) variant engineered to improve binding to its natural receptors, BCMA and TACI (90-92). Finally, approaches using CAR T cells as a platform to recruit a broader immune infiltrate are promising (93), as T cells can be engineered to secrete immune checkpoint-blocking scFvs or bispecific $\mathrm{T}$ cell engagers (Figure $3 \mathrm{E}$ ) to allow local immune targeting within the tumor microenvironment of an otherwise broadly-expressed antigen without systemic toxicity (94).

\section{Concluding remarks}

The field of CAR T cell therapy is growing rapidly. We undertook this Review with the thought of covering all of CAR T cell therapy for hematologic malignancies, but then found that there were enormous progress and data to cover even within the limited scope of B cell malignancies: from variations in CAR molecular design; to clinical observations describing efficacy with multiple CD19-directed CAR T cell products, their unpredicted but relatively similar toxicities, and how systems had to be devised to measure and quantify and report them; to correlations and mechanisms discovered in the clinic and then modeled in vitro and in animals to discover and test pathways and druggable targets. Not only is CAR T cell therapy transformative in its potential to treat cancer with a single infusion, but its complex, cellular nature and its role in directing other cell-cell interactions, whether between CAR T cell and tumor cell or endothelial cell or macrophage, lend themselves to deep scientific probing and manipulation of its functions.

\section{Acknowledgments}

MJF was supported by the NIH/National Cancer Institute (K12CA087723).

Address correspondence to: Marcela V. Maus, Massachusetts General Hospital, 149 13th Street, Room 3.216, Charlestown, Massachusetts 02129, USA. Email: mvmaus@mgh.harvard.edu. 
1. Kochenderfer JN, et al. Construction and preclinical evaluation of an anti-CD19 chimeric antigen receptor. JImmunother. 2009;32(7):689-702.

2. Milone MC, et al. Chimeric receptors containing CD137 signal transduction domains mediate enhanced survival of T cells and increased antileukemic efficacy in vivo. Mol Ther. 2009;17(8):1453-1464

3. Maus MV, Grupp SA, Porter DL, June CH. Antibody-modified T cells: CARs take the front seat for hematologic malignancies. Blood. 2014;123(17):2625-2635.

4. Porter DL, Levine BL, Kalos M, Bagg A, June $\mathrm{CH}$. Chimeric antigen receptor-modified T cells in chronic lymphoid leukemia. $N$ Engl J Med. 2011;365(8):725-733.

5 . Brentjens RJ, et al. Safety and persistence of adoptively transferred autologous CD19targeted $\mathrm{T}$ cells in patients with relapsed or chemotherapy refractory B-cell leukemias. Blood. 2011;118(18):4817-4828.

6. Kochenderfer JN, et al. Eradication of B-lineage cells and regression of lymphoma in a patient treated with autologous $\mathrm{T}$ cells genetically engineered to recognize CD19. Blood. 2010;116(20):4099-4102.

7. Locke FL, et al. Phase 1 results of ZUMA-1: a multicenter study of KTE-C19 anti-CD19 CAR T cell therapy in refractory aggressive lymphoma. Mol Ther. 2017;25(1):285-295.

8. Schuster SJ, et al. Tisagenlecleucel in adult relapsed or refractory diffuse large B-cell lymphoma. N Engl J Med. 2019;380(1):45-56.

9. Maude SL, et al. Tisagenlecleucel in children and young adults with B-cell lymphoblastic leukemia. N Engl J Med. 2018;378(5):439-448.

10. Lee DW, et al. ASTCT consensus grading for cytokine release syndrome and neurologic toxicity associated with immune effector cells. Biol Blood Marrow Transplant. 2019;25(4):625-638.

11. [No authors listed.] JCAR015 in ALL: a rootcause investigation. Cancer Discov. 2018;8(1):4-5.

12. Kalos M, et al. T cells with chimeric antigen receptors have potent antitumor effects and can establish memory in patients with advanced leukemia. Sci Transl Med. 2011;3(95):95ra73.

13. Grupp SA, et al. Chimeric antigen receptormodified $\mathrm{T}$ cells for acute lymphoid leukemia. NEngl J Med. 2013;368(16):1509-1518.

14. Riegler LL, Jones GP, Lee DW. Current approaches in the grading and management of cytokine release syndrome after chimeric antigen receptor T-cell therapy. Ther Clin Risk Manag. 2019;15:323-335.

15. Khadka RH, Sakemura R, Kenderian SS, Johnson AJ. Management of cytokine release syndrome: an update on emerging antigen-specific T cell engaging immunotherapies. Immunotherapy. 2019;11(10):851-857.

16. Lee DW, et al. Current concepts in the diagnosis and management of cytokine release syndrome. Blood. 2014;124(2):188-195.

17. Porter D, Frey N, Wood PA, Weng Y, Grupp SA. Grading of cytokine release syndrome associated with the CAR T cell therapy tisagenlecleucel. J Hematol Oncol. 2018;11(1):35.

18. Maude SL, et al. Chimeric antigen receptor $\mathrm{T}$ cells for sustained remissions in leukemia. N Engl J Med. 2014;371(16):1507-1517.
19. Lee DW, et al. T cells expressing CD19 chimeric antigen receptors for acute lymphoblastic leukaemia in children and young adults: a phase 1 dose-escalation trial. Lancet. 2015;385(9967):517-528.

20. Davila ML, et al. Efficacy and toxicity management of 19-28z CAR T cell therapy in B cell acute lymphoblastic leukemia. Sci Transl Med. 2014;6(224):224ra25.

21. Turtle CJ, et al. CD19 CAR-T cells of defined CD4+:CD8+ composition in adult B cell ALL patients. J Clin Invest. 2016;126(6):2123-2138.

22. Frigault MJ, June $\mathrm{CH}$. Predicting cytokine storms: it's about density. Blood. 2011;118(26):6724-6726.

23. Chatenoud L, et al. In vivo cell activation following OKT3 administration. Systemic cytokine release and modulation by corticosteroids. Transplantation. 1990;49(4):697-702.

24. Brudno JN, Kochenderfer JN. Toxicities of chimeric antigen receptor $\mathrm{T}$ cells: recognition and management. Blood. 2016;127(26):3321-3330.

25. Gattinoni L, et al. Removal of homeostatic cytokine sinks by lymphodepletion enhances the efficacy of adoptively transferred tumor-specific CD8+ T cells. J Exp Med. 2005;202(7):907-912.

26. Klebanoff CA, Khong HT, Antony PA, Palmer DC, Restifo NP. Sinks, suppressors and antigen presenters: how lymphodepletion enhances $\mathrm{T}$ cell-mediated tumor immunotherapy. Trends Immunol. 2005;26(2):111-117.

27. Raje N, et al. Anti-BCMA CAR T-cell therapy bb2121 in relapsed or refractory multiple myeloma. NEngl JMed. 2019;380(18):1726-1737.

28. Gust J, et al. Endothelial activation and bloodbrain barrier disruption in neurotoxicity after adoptive immunotherapy with CD19 CAR-T cells. Cancer Discov. 2017;7(12):1404-1419.

29. Frigault MJ, et al. Tisagenlecleucel CAR T-cell therapy in secondary CNS lymphoma. Blood. 2019;134(11):860-866.

30. Locke FL, et al. Preliminary results of prophylactic tocilizumab after axicabtageneciloleucel (axi-cel; KTE-C19) treatment for patients with refractory, aggressive non-Hodgkin lymphoma (NHL). Blood. 2017;130(suppl 1):1547.

31. Karschnia P, et al. Clinical presentation, management, and biomarkers of neurotoxicity after adoptive immunotherapy with CAR T cells. Blood. 2019;133(20):2212-2221.

32. Taraseviciute A, et al. Chimeric antigen receptor $\mathrm{T}$ cell-mediated neurotoxicity in nonhuman primates. Cancer Discov. 2018;8(6):750-763.

33. Lowe KL, Mackall CL, Norry E, Amado R, Jakobsen BK, Binder G. Fludarabine and neurotoxicity in engineered T-cell therapy. Gene Ther. 2018;25(3):176-191.

34. Zabernigg A, Maier H, Thaler J, Gattringer C. Late-onset fatal neurological toxicity of fludarabine. Lancet. 1994;344(8939-8940):1780.

35. Cheson BD, Vena DA, Foss FM, Sorensen JM. Neurotoxicity of purine analogs: a review. JClin Oncol. 1994;12(10):2216-2228.

36. Gandhi V, Plunkett W. Cellular and clinical pharmacology of fludarabine. Clin Pharmacokinet. 2002;41(2):93-103.

37. Adjei AA, Dagnino L, Wong MM, Paterson AR. Protection against fludarabine neurotoxicity in leukemic mice by the nucleoside transport inhib- itor nitrobenzylthioinosine. Cancer Chemother Pharmacol. 1992;31(1):71-75.

38. Shimabukuro-Vornhagen A, et al. Cytokine release syndrome. JImmunother Cancer. 2018;6(1):56

39. Teachey DT, et al. Cytokine release syndrome after blinatumomab treatment related to abnormal macrophage activation and ameliorated with cytokine-directed therapy. Blood. 2013;121(26):5154-5157.

40. Teachey DT, et al. Identification of predictive biomarkers for cytokine release syndrome after chimeric antigen receptor T-cell therapy for acute lymphoblastic leukemia. Cancer Discov. 2016;6(6):664-679.

41. Shultz LD, et al. Multiple defects in innate and adaptive immunologic function in NOD/LtSzscid mice. JImmunol. 1995;154(1):180-191.

42. Giavridis T, van der Stegen SJC, Eyquem J, Hamieh M, Piersigilli A, Sadelain M. CAR T cell-induced cytokine release syndrome is mediated by macrophages and abated by IL-1 blockade. Nat Med.2018;24(6):731-738.

43. Norelli M, et al. Monocyte-derived IL-1 and IL-6 are differentially required for cytokine-release syndrome and neurotoxicity due to CAR T cells. Nat Med. 2018;24(6):739-748.

44. Sterner RM, et al. GM-CSF inhibition reduces cytokine release syndrome and neuroinflammation but enhances CAR-T cell function in xenografts. Blood. 2019;133(7):697-709.

45. Porter DL, et al. Chimeric antigen receptor $\mathrm{T}$ cells persist and induce sustained remissions in relapsed refractory chronic lymphocytic leukemia. Sci Transl Med. 2015;7(303):303ra139.

46. Park JH, et al. Long-term follow-up of CD19 CAR therapy in acute lymphoblastic leukemia. $N$ Engl J Med. 2018;378(5):449-459.

47. Neelapu SS, et al. Chimeric antigen receptor T-cell therapy - assessment and management of toxicities. Nat Rev Clin Oncol. 2018;15(1):47-62.

48. Mueller KT, et al. Cellular kinetics of CTL019 in relapsed/refractory B-cell acute lymphoblastic leukemia and chronic lymphocytic leukemia. Blood. 2017;130(21):2317-2325.

49. Grupp SA, et al. Updated analysis of the efficacy and safety of tisagenlecleucel in pediatric and young adult patients with relapsed/refractory (R/R) acute lymphoblastic leukemia. Blood. 2018;132(suppl 1):895.

50. Sotillo E, et al. Convergence of acquired mutations and alternative splicing of CD19 enables resistance to CART-19 immunotherapy. Cancer Discov. 2015;5(12):1282-1295.

51. Ruella M, et al. Induction of resistance to chimeric antigen receptor $\mathrm{T}$ cell therapy by transduction of a single leukemic B cell. Nat Med. 2018;24(10):1499-1503.

52. Turtle CJ, et al. Immunotherapy of nonHodgkin's lymphoma with a defined ratio of CD8+ and CD4+ CD19-specific chimeric antigen receptor-modified T cells. Sci Transl Med. 2016;8(355):355ra116.

53. Maude SL, et al. Efficacy of humanized CD19-targeted chimeric antigen receptor (CAR)-modified $\mathrm{T}$ cells in children and young adults with relapsed/refractory acute lymphoblastic leukemia. Blood. 2016;128(22):217. 
54. Brudno JN, et al. Safety and feasibility of antiCD19 CAR T cells with fully human binding domains in patients with B-cell lymphoma. Nat Med. 2020;26(2):270-280.

55. Neelapu SS, et al. Axicabtagene ciloleucel CAR T-cell therapy in refractory large B-cell lymphoma. NEngl JMed. 2017;377(26):2531-2544.

56 . Locke FL, et al. Long-term safety and activity of axicabtagene ciloleucel in refractory large B-cell lymphoma (ZUMA-1): a single-arm, multicentre, phase 1-2 trial. Lancet Oncol. 2019;20(1):31-42.

57. El-Galaly TC, et al. Routine imaging for diffuse large B-cell lymphoma in first complete remission does not improve post-treatment survival: a Danish-Swedish population-based study. J Clin Oncol. 2015;33(34):3993-3998.

58. Neelapu SS, et al. CD19-loss with preservation of other B cell lineage features in patients with large B cell lymphoma who relapsed post-axi-cel. Blood. 2019;134(suppl 1):203.

59. Nastoupil LJ, et al. Axicabtagene ciloleucel (axi-cel) CD19 chimeric antigen receptor (CAR) $\mathrm{T}$-cell therapy for relapsed/refractory large B-cell lymphoma: real world experience. Blood. 2018;132(suppl 1):91.

60. Jacobson CA, et al. Axicabtagene ciloleucel in the real world: outcomes and predictors of response, resistance and toxicity. Blood. 2018;132(suppl 1):92.

61. Neelapu SS, et al. Axicabtagene ciloleucel CAR T-cell therapy in refractory large B-cell lymphoma. NEngl JMed. 2017;377(26):2531-2544.

62. Rossi J, et al. Preinfusion polyfunctional antiCD19 chimeric antigen receptor T cells are associated with clinical outcomes in NHL. Blood. 2018;132(8):804-814.

63. Hill BT, Roberts ZJ, Xue A, Rossi JM, Smith MR. Rapid tumor regression from PD-1 inhibition after anti-CD19 chimeric antigen receptor T-cell therapy in refractory diffuse large B-cell lymphoma [published online August 30, 2019]. Bone Marrow Transplant. https://doi.org/10.1038/ s41409-019-0657-3.

64. Oak J, et al. Target antigen downregulation and other mechanisms of failure after axicabtagene ciloleucel (CAR19) therapy. Blood. 2018;132(suppl 1):4656.

65. Schuster SJ. CD19-directed CAR T cells gain traction. Lancet Oncol. 2019;20(1):2-3.

66. Crump M, et al. Outcomes in refractory diffuse large B-cell lymphoma: results from the international SCHOLAR-1 study. Blood. 2017;130(16):1800-1808.

67. Bishop MR, et al. Tisagenlecleucel in relapsed/ refractory diffuse large B-cell lymphoma patients without measurable disease at infusion. Blood Adv. 2019;3(14):2230-2236.

68. Sommermeyer D, et al. Chimeric antigen receptor-modified $\mathrm{T}$ cells derived from defined CD8+ and CD4+ subsets confer superior antitumor reac- tivity in vivo. Leukemia. 2016;30(2):492-500.

69. Abramson JS, et al. Pivotal safety and efficacy results from Transcend NHL 001, a multicenter phase 1 study of lisocabtagene maraleucel (lisocel) in relapsed/refractory (R/R) large B cell lymphomas. Paper presented at: American Society of Hematology Annual Meeting; December 7-10, 2019; Orlando, Florida, USA.

70. van Imhoff GW, et al. Ofatumumab versus rituximab salvage chemoimmunotherapy in relapsed or refractory diffuse large B-cell lymphoma: the ORCHARRD study. JClin Oncol. 2017;35(5):544-551.

71. Kebriaei P, et al. Phase I trials using Sleeping Beauty to generate CD19-specific CAR T cells. JClin Invest. 2016;126(9):3363-3376.

72. Wang $X$, et al. Phase 1 studies of central memoryderived CD19 CAR T-cell therapy following autologous HSCT in patients with B-cell NHL. Blood. 2016;127(24):2980-2990.

73. Sauter CS, et al. CD19 CAR T cells following autologous transplantation in poor-risk relapsed and refractory B-cell non-Hodgkin lymphoma. Blood. 2019;134(7):626-635.

74. Wang ML, et al. KTE-X19, an anti-CD19 chimeric antigen receptor (CAR) $\mathrm{T}$ cell therapy, in patients (pts) with relapsed/refractory $(\mathrm{R} / \mathrm{R})$ mantle cell lymphoma (MCL): results of the phase 2 ZUMA-2 study. Biol Blood Marrow Transplant. 2019;26(3):S1.

75. Jacobson CA, et al. End of phase 1 results from Zuma-6: axicabtagene ciloleucel (axi-cel) in combination with atezolizumab for the treatment of patients with refractory diffuse large $B$ cell lymphoma. Biol Blood Marrow Transplant. 2019;25(3):S173.

76. Singh N, et al. Impaired death receptor signaling in leukemia causes antigen-independent resistance by inducing CAR T cell dysfunction [published online January 30, 2020]. Cancer Discov. https://doi.org/10.1158/2159-8290.cd-19-0813.

77. Fraietta JA, et al. Determinants of response and resistance to CD19 chimeric antigen receptor (CAR) T cell therapy of chronic lymphocytic leukemia. Nat Med. 2018;24(5):563-571.

78. Fraietta JA, et al. Ibrutinib enhances chimeric antigen receptor T-cell engraftment and efficacy in leukemia. Blood. 2016;127(9):1117-1127.

79. Finney OC, et al. Toward durable multiple myeloma regressions with anti-BCMA CAR T cells with PI3K inhibition and reduced time of culture. Molecular Therapy. 2019;27(4 suppl 1):171.

80. Benjamin R, et al. Preliminary data on safety, cellular kinetics and anti-leukemic activity of UCART19, an allogeneic anti-CD19 CAR T-cell product, in a pool of adult and pediatric patients with high-risk CD19+ relapsed/refractory B-cell acute lymphoblastic leukemia. Blood. 2018;132(suppl 1):896.

81. Orlando EJ, et al. Genetic mechanisms of target antigen loss in CAR19 therapy of acute lymphoblastic leukemia. Nat Med.2018;24(10):1504-1506.

82. Gardner R, et al. Acquisition of a CD19-negative myeloid phenotype allows immune escape of MLL-rearranged B-ALL from CD19 CAR-T-cell therapy. Blood. 2016;127(20):2406-2410.

83. Hamieh M, et al. CAR T cell trogocytosis and cooperative killing regulate tumour antigen escape. Nature. 2019;568(7750):112-116.

84. Ruella M, et al. Dual CD19 and CD123 targeting prevents antigen-loss relapses after CD19-directed immunotherapies. J Clin Invest. 2016;126(10):3814-3826.

85. Savoldo B, et al. CD28 costimulation improves expansion and persistence of chimeric antigen receptor-modified $\mathrm{T}$ cells in lymphoma patients. JClin Invest. 2011;121(5):1822-1826.

86. Shah NN, et al. Results of a phase I study of bispecific anti-CD19, anti-CD20 chimeric antigen receptor (CAR) modified T cells for relapsed, refractory, non-Hodgkin lymphoma. J Clin Oncol. 2019;37(15 suppl):2510.

87. Ormhøj M, et al. Chimeric antigen receptor T cells targeting CD79b show efficacy in lymphoma with or without cotargeting CD19. Clin Cancer Res. 2019;25(23):7046-7057.

88. Schultz LM, et al. Phase 1 study of CD19/CD22 bispecific chimeric antigen receptor (CAR) therapy in children and young adults with $\mathrm{B}$ cell acute lymphoblastic leukemia (ALL). Blood. 2018;132(suppl 1):898.

89. Scarfò I, et al. Anti-CD37 chimeric antigen receptor $\mathrm{T}$ cells are active against $\mathrm{B}$ - and $\mathrm{T}$-cell lymphomas. Blood. 2018;132(14):1495-1506.

90. Lee L, et al. An APRIL-based chimeric antigen receptor for dual targeting of BCMA and TACI in multiple myeloma. Blood. 2018;131(7):746-758.

91. Schmidts A, et al. Rational design of a trimeric APRIL-based CAR-binding domain enables efficient targeting of multiple myeloma. Blood Adv. 2019;3(21):3248-3260.

92. Brown CE, et al. Regression of glioblastoma after chimeric antigen receptor T-cell therapy. $N$ Engl J Med. 2016;375(26):2561-2569.

93. Yeku OO, Purdon TJ, Koneru M, Spriggs D, Brentjens RJ. Armored CAR T cells enhance antitumor efficacy and overcome the tumor microenvironment. Sci Rep. 2017;7(1):10541.

94. Choi BD, et al. CAR-T cells secreting BiTEs circumvent antigen escape without detectable toxicity. Nat Biotechnol. 2019;37(9):1049-1058.

95. Bachanova V, et al. Correlative analyses of cytokine release syndrome and neurological events in tisagenlecleucel-treated relapsed/refractory diffuse large B-cell lymphoma patients. Paper presented at: 15th International Conference on Malignant Lymphoma; June 18-22, 2019; Lugano, Switzerland.http://www.lymphcon.ch/icml/website/ doc/15-ICML_Abstract_Book.pdf. Accessed February 5,2020 